\title{
HISTORIA AMBIENTAL DE LA LAGUNA SECA, TARTAGAL SALTA, NOROESTE ARGENTINO
}

\author{
PAMELA TATIANA FIERRO, JULIO JOSE KULEMEYER \\ Facultad de Ingeniería, INECOA CONICET/UNJu, Avenida Italo Palanca No 10, CP 4600, \\ S.S. de Jujuy, Argentina.pamelatatianafierro@gmail.com,jjkulemeyer@fi.unju.edu.ar \\ LILIANA CONCEPCIÓN LUPO \\ Laboratorio de Palinología, INECOA CONICET/UNJu, Alberdi No 47, \\ CP4600, S.S. de Jujuy, Argentina.lupolc@yahoo.com.ar

\section{SANTIAGO GIRALT} \\ Instituto de Ciencias de la Tierra Jaume Almera (ICTJA-CSIC), C/Solé y Sabarís S/N, 08028, \\ Universidad de Barcelona, España.sgiralt@ictja.csic.es
}

\begin{abstract}
ENVIRONMENTAL HISTORY OF LAGUNA SECA, TARTAGAL SALTA, NORTHWEST ARGENTINA. First results from an interdisciplinary research on the history of landscape in the mountain range of Tartagal, Province of Salta (NW Argentina) for the 1959/2012 period are given. Geochemical, mineralogical and palynological studies with high temporal resolution have been performed in sediments of a core from Laguna Seca (22 $21^{\prime} 1.81^{\prime \prime}$ S, 6352'10.90"W). Dentations by ${ }^{210} \mathrm{~Pb}$ show that the sedimentary record comprises 53 years. Sediments are composed of reddish massive clays and silts, with interbedded clays rich in organic matter and fibrous plants macro remains in the base of the core. Presence of quartz, illite and clinochlorite is prominent; most relevant geochemical components are $\mathrm{Al}, \mathrm{Si}$ and $\mathrm{K}$. Redundancy analysis indicates that most of chemical elements are associated to terrigenous minerals (quartz, albite and illite). Pollen types found belong to the Yungas forest, like Alnus acuminata, Anadenanthera colubrina, Celtis sp. and Schinus sp. Geochemical, mineralogical and palynological results are consistent with the annual precipitation in Tartagal during last decades, and reflect some correlation with phenomena of mass movement as well as landslides occurred in the mountain range of Tartagal and its oriental piedmont. The multiproxies data integration has generated the basis for reconstructing changes in vegetation, climate and history of anthropogenic disturbance during the Holocene in Subandine Sierra the Northwest Argentine.
\end{abstract}

Key words: historical reconstruction, multiproxies, late Holocene, catastrophic events, Yungas.

RESUMO - São apresentados os resultados de uma pesquisa interdisciplinar sobre a história da paisagem na Serrania de Tartagal, Salta, para o período de 1959-2012 AD. Estudos de alta resolução temporal incluindo geoquímica, mineralogia e palinologia foram realizados em sedimentos de um testemunho da Laguna Seca (22²1'1,81'S, 6352’10,90”O). As datações mostram que o registo sedimentar compreendem um total de 53 anos. Os sedimentos são compostos de argila avermelhada maciça e silte, com intercalações de argilas ricas em matéria orgânica e macro-restos vegetais fibrosos na base. A presença de quartzo, ilita e clinoclorita é destacada; os componentes geoquímicos mais importantes são Al, Si e K. A análise de redundância indica que a maioria dos elementos químicos estão associados com minerais terrígenos (quartzo, albita e ilita). Os tipos polínicos encontrados correspondem à Floresta de Yungas como Alnus acuminata, Anadenanthera colubrina, Celtis sp. e Schinus sp. Os resultados de geoquímica, mineralogia e palinologia são consistentes com a precipitação anual em Tartagal nas últimas décadas e refletem uma correlação com os fenômenos das inundações e deslizamentos de terra na região montanhosa de Tartagal. A integração de dados multiproxis gerou as bases para reconstruir as mudanças na vegetação, clima e história de perturbação antrópica durante o Holoceno nas Serras Subandinas do noroeste argentino.

Palavras-chave: reconstrução histórica, multiproxis, Holoceno superior, eventos catastróficos, Yungas. 


\section{INTRODUCCIÓN}

En esta contribución, se presentan los primeros resultados de las investigaciones interdisciplinarias destinadas a reconstruir la historia del paisaje y el clima en el sector norte de las Sierras Subandinas del Noroeste Argentino, durante los últimos 53 años, mediante el estudio de alta resolución temporal de un testigo sedimentario de una laguna somera. El objetivo es aportar información que ayude a la comprensión de las condiciones desencadenantes de eventos catastróficos y las potenciales consecuencias para la población e infraestructura de la región.

Durante el siglo XX, se ha observado un aumento de eventos catastróficos (inundaciones, sequias, aluvionamientos, entre otros), simultáneos a un incremento de la temperatura y el cambio de régimen de lluvias. Por lo tanto, con el fin de intentar mejorar la predictibilidad de estos eventos y comprender cuales son las principales causas que los desencadenan, se pueden utilizar los registros naturales, en este caso, los sedimentos depositados en el fondo de las lagunas, como archivos ambientales detallados de estos sucesos.

Se conocen diversos antecedentes de trabajos interdisciplinarios utilizando multiproxies para el Holoceno en los Andes Centrales, particularmente en el Noroeste Argentino (Lupo, 1998; Kulemeyer \& Lupo, 1998; Kulemeyer et al., 1999; Schäbitz et al., 2001; Kulemeyer, 2005; Torres, 2010; Cortes, 2010; Bonachea et al., 2010; Yacobaccio et al., 2013). Metodológicamente, se puede destacar el trabajo de Lupo et al. (2006), en un sector boscoso de Yungas, mientras que los antecedentes de estudios con alta resolución para el último siglo en zonas boscosas del Amazonia de Brasil y Bolivia son escasos (Villagrán, 1993; Argollo \& Mourguiart, 2000; Burbridge et al., 2004; May, 2007; May et al., 2008; Bonachea et al., 2010; Behling, 2011; Mayle, 2011; May, 2011).

Durante el Holoceno tardío la acción antrópica sobre los recursos naturales fue creciente y la respuesta de los sistemas de montaña se manifestó como una generalizada degradación regional, con intensificación de los procesos de erosión y acumulación (Brown, 1995; Castro, 1995; Grau et al., 2005; Kulemeyer, 2013). El extremo oeste del Departamento San Martín, Salta, es un área de gran inestabilidad territorial y susceptible a la erosión. Por este motivo, ofrece un escenario óptimo para el estudio de eventos naturales catastróficos en un escenario muy sensible. En los últimos años hubo aluvionamientos de importantes dimensiones que afectaron a la ciudad de Tartagal en el verano de 2005-2006 y en febrero de 2009, observándose una gran vulnerabilidad a los eventos extremos (Cabral \& Plaza, 2006; Baumman et al., 2009; Cardozo, 2013). Los fenómenos hidrológicos que se presentan en la cuenca del río Tartagal, se vinculan principalmente a procesos torrenciales y de remoción en masa (Novillo \& Sarandón, 2002; Cristobal et al., 2009).

En la Sierra de Tartagal son numerosas las cicatrices de deslizamientos en los faldeos de los cordones montañosos. Estos disturbios se caracterizan por la remoción completa del suelo y material vegetal, creando ambientes caracterizados por una alta irradiación, baja fertilidad orgánica del suelo y mayores oscilaciones de temperatura y humedad, donde, una vez producido el deslizamiento, el sector afectado es colonizado por nuevas especies vegetales, lográndose cierto grado de estabilización (Veblen \& Asthon, 1978; Garwood et al., 1979; Guariguata, 1990; Blodgett, 1998; Gonzalez Bonorino et al., 2001; Trauth et al., 2003; Remondo et al., 2005; Cristóbal et al., 2009; Paolini, 2014). La mayor parte de los deslizamientos observados en la región son naturales; una cantidad menor está causada por la desestabilización de laderas vinculada al emplazamiento de caminos u otras obras de infraestructura realizadas para distintas actividades extractivas de la zona (Grau \& Brown, 1995; Gonzalez Bonorino et al., 2001; Paolini et al., 2001; Cristóbal et al., 2009).

En esta investigación se presentan el análisis de tres proxies: sedimentología, geoquímica y palinología, con una cronología de alta resolución para el periodo 1959-2012, los cuales se vinculan a datos de precipitación y eventos catastrófico aluvionales, como primeros datos de la historia ambiental de la Laguna Seca de Tartagal, en el Noroeste Argentino.

\section{ÁREA DE ESTUDIO}

\section{Localización}

El área de estudio (Figuras 1A-B) comprende la cuenca alta del río Tartagal, desde su nacimiento en la Sierra homónima (1300 msnm), hasta su desembocadura en el piedemonte, en las inmediaciones de la localidad de Tartagal y corresponde al departamento San Martín, al norte de la Provincia de Salta, Argentina.

\section{Geología}

La Sierra de Tartagal presenta un anticlinal asimétrico, de rumbo submeridiano y buzante hacia el sur. El núcleo del mismo aflora en la transición de la cuenca media a alta del río Tartagal y expone sedimentitas competentes del Paleozoico Superior. El flanco oriental presenta afloramientos de la Formación Tranquitas, de edad Mioceno temprano a medio, constituida por areniscas, areniscas calcáreas y pelitas medianamente competentes de colores grises a verdosos. La alta cuenca del río Tartagal y sus nacientes se encuentran sobre el flanco occidental del anticlinal, donde los afloramientos son continuos y completan la columna estratigráfica con el Terciario Subandino (formaciones Tariquía, Guandacay y Emboruzú), integrados por areniscas conglomerádicas, areniscas medianas y pelitas con alto grado de erodabilidad y coloración general rojiza. Las areniscas limosas y pelitas subordinadas del Terciario Subandino cubren la mayor parte de la cuenca, con buzamientos de hasta $40^{\circ}$; las máximas pendientes se localizan en los grupos Cuevo y Tacurú, así como también en la Formación Tranquitas (Gonzalez Bonorino et al., 2001; Cardozo, 2013).

El sitio específico de nuestro estudio es la Laguna Seca $\left(22^{\circ} 21^{\prime} 1,81^{\prime \prime} \mathrm{S}, 63^{\circ} 52^{\prime} 10,90^{\prime \prime} \mathrm{O}\right)$ (Figura 1C), ubicada a 
780 msnm y originada a partir de un deslizamiento durante el Holoceno, en la cabecera del río Tartagal. Localmente, el sustrato está constituido principalmente por rocas del Terciario plegadas y friables, que alternan con sedimentitas del Carbonífero y Pérmico, más resistentes a la erosión.

\section{Vegetación y clima}

Los Bosques Andinos Subtropicales o Yungas (Figura 1A), también denominados en la literatura "Selva TucumanoBoliviana" o "Selva Tucumano-Oranense", se distribuyen en Argentina desde la frontera con Bolivia (22 $\left.{ }^{\circ} \mathrm{S}\right)$ hasta el Norte de la Provincia de Catamarca $\left(29^{\circ} \mathrm{S}\right)$, pasando por las provincias de Salta, Jujuy y Tucumán. Se caracterizan por un fuerte gradiente altitudinal, lo que da lugar a que la vegetación se organice en pisos o franjas de vegetación de características fisonómicas y florísticas bien diferenciadas: Bosque Montano, Selva Montana y Selva Pedemontana. El Bosque Montano representa el piso ecológico de los "bosques nublados" propiamente dichos, entre los 1500 y $3000 \mathrm{msnm}$, con precipitaciones de $800 \mathrm{~mm} /$ año. Se encuentra próximo a los pastizales de neblina y muestra un paisaje heterogéneo, con bosques en distintos estadios sucesionales. Las especies dominantes son: Podocarpus parlatorei Pilg., Alnus acuminata Kunth, Juglans australis Griseb, Viburnum seemanii Graebn, Roupala meisneri Sleumer, Fuchsia boliviana Carrière, Ilex argentinum A.St.-Hil. y Sambucus peruviana Kunth.

La Selva Montana representa la franja altitudinal de máximas precipitaciones pluviales (hasta $2000 \mathrm{~mm} / \mathrm{año}$ ). Ocupa las laderas de las montañas entre los 800 y 1500 msnm. Entre las especies dominantes se puede señalar a Ficus maroma A. Cast., Cinnamomum porphyrium (Griseb.) Kosterm, Nectandra pichurim Kunth, Ocotea puberula (Rich.) Nees, Inga edulis Mart., I. marginata Willd, I. saltensis Burkart, y Blepharocalix salicifolius (Kunth) O.Berg. (Brown, 1995; Brown et al., 2001; Grau, 2004).
A

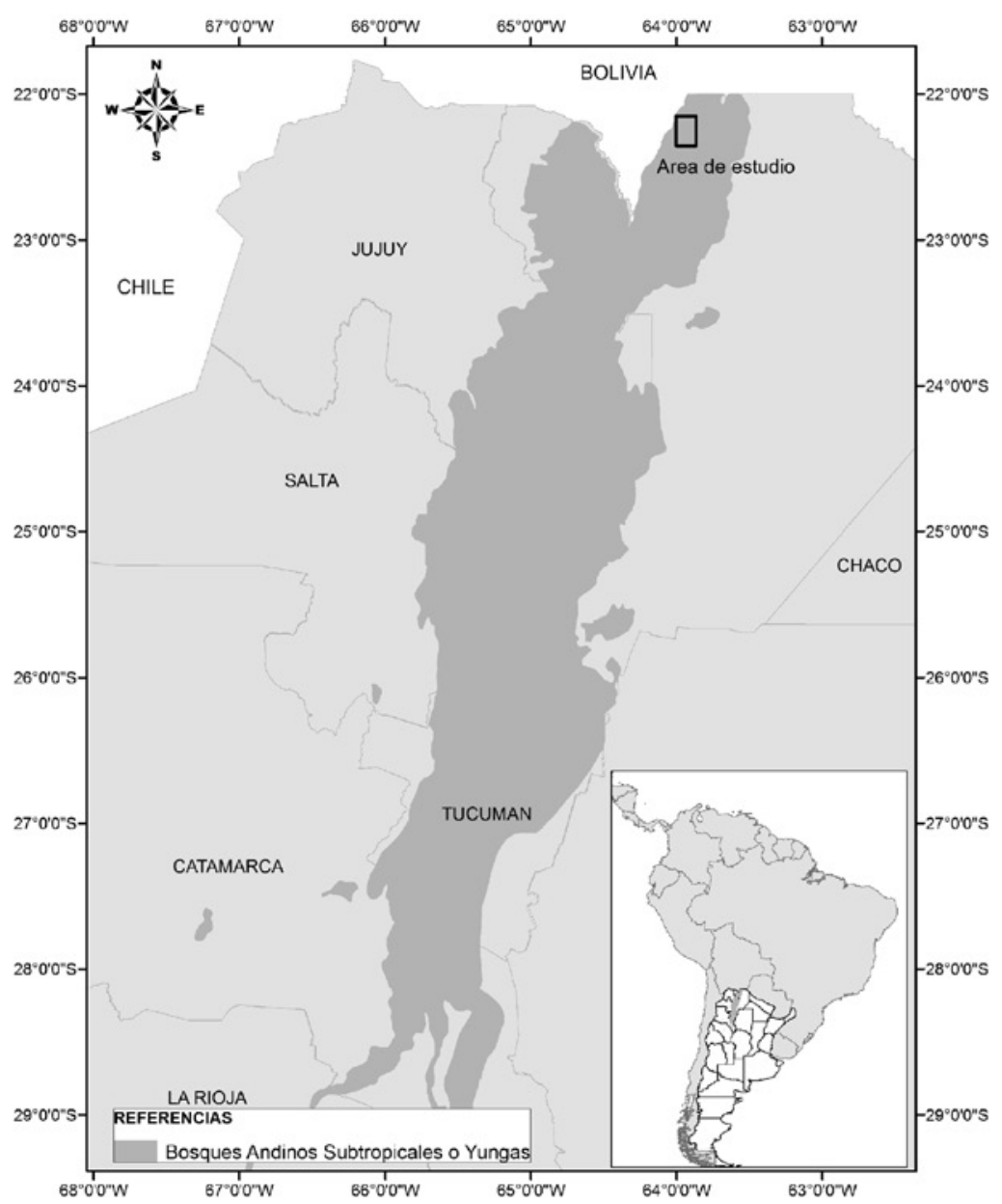

B

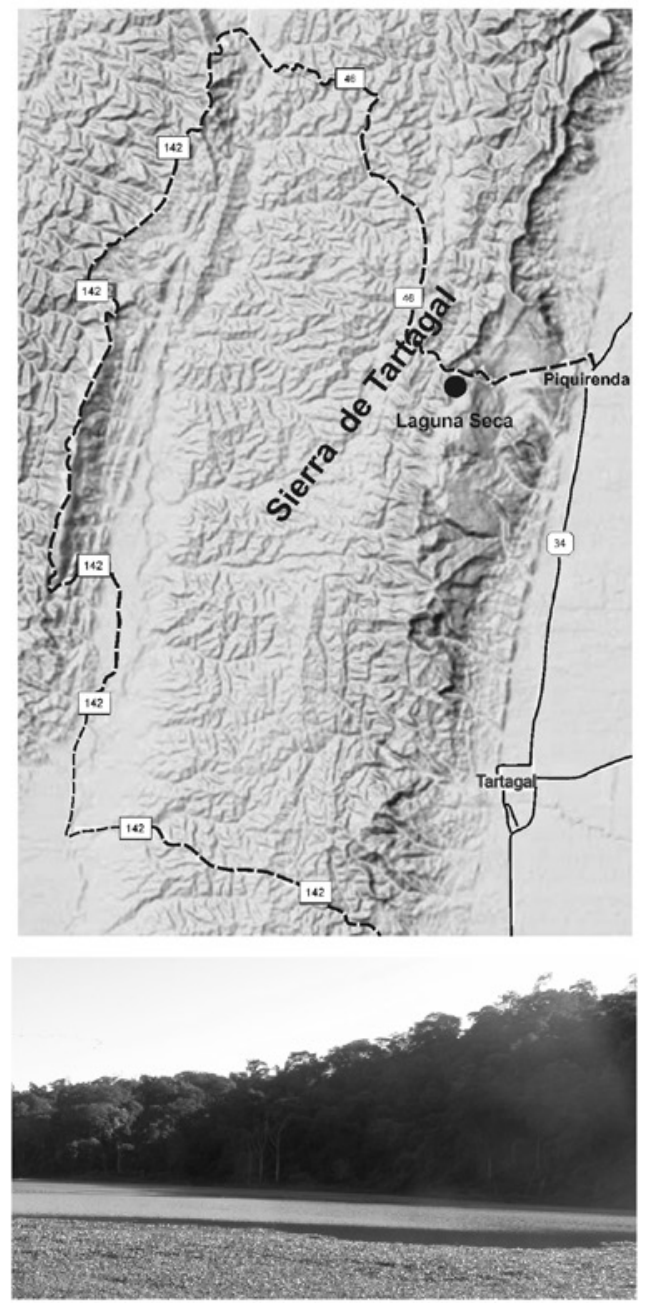

Figura 1. A, distribución de los Bosques Andinos Subtropicales o Yungas en Argentina y localización del área de estúdio; B-C, área de estudio, localización y fotografía de la Laguna Seca en la Sierra de Tartagal.

Figure 1. A, distribution of Andean Subtropical Forests or Yungas in Argentina and location of the study area; B-C, study area, location and photography of Laguna Seca in the mountain range of Tartagal. 
La Sierra de Tartagal (Figura 1B) se encuentra ocupada mayormente por la Selva Pedemontana, el piso ecológico de las Yungas con mayor presión de transformación y degradación (Brown, 1995; Castro, 1995; Brown et al., 2001; Grau et al., 2005). Ocupan el pedemonte y las laderas de las montañas bajas entre los 400 y $800 \mathrm{msnm}$, con precipitaciones de $1000 \mathrm{~mm} /$ año La vegetación arbórea dominante está caracterizada Anadenanthera colubrina (Vell.) Brenan, Calycophylum multiflorum Griseb., Phyllostylon rhamnoides Poisson., Cordia trichotoma (Vell.) Arráb. ex Steud, Tabebuia impetiginosa (Mart. ex DC.) Mattos, Enterolobium contortisiliquum (Vell.) Morong, Patagonula americana L. Gottschling \& J.S. Mill, Myroxylon peruiferum L. F. y Astronium urundeuva (Fr. All.) Engl., acompañada de numerosos arbustos como Urera baccifera (L.) Gaudich, Buddleja stachyoides Cham. \& Schltdl, Erythroxylum argentinum Schulz, Cestrum lorentzianum Griseb, Tecoma stans (L.) H.B.K., Lessingianthus plantaginoides (Less.) H. Rob., Chamissoa altissima H. B. K., Pogonopus tubulosus (A. Rich. in DC.) K. Schum, Senna bicapsularis, Cnicothamnus lorentzii Griseb, Porlieria microphylla (Baill.) Descole, O'Donell \& Lourteig, etc. hierbas: Nicotiana sylvestris Speg. \& Comes, Justicia comata (L.) Lam., Liabum polymnioides R.E. Fr., Justicia goudotti V.A.W. Graham, Lobelia xalapensis Kunth, Geophila repens (L.) I. M. Johnst., Onoseris alata Rusby, Ruellia geminiflora Kunth, Syphocampilus aureus, Pseudelephantopus spiralis (Less.) Cronquist, Setaria sp., Panicum sp., etc. lianas: Schubertia schreiteri Descole \& T. Mey, Dolichandra unguis-cati (L.) L.G. Lohmann, Pseudogynoxys benthami Cabrera etc. y epifitas Oncidium herzogii Schltr., Catasetum sp., Campylocentrum grisebachii Cogn., Polypodium sp., Tillandsia sp., etc. (Cabrera, 1976; Prado, 1995; Brown, 1995; Blundo \& Malizia, 2009).

El clima es de carácter monzónico, determinado por dos centros de alta presión: el Anticiclón Subtropical Atlántico del Sur que aporta vientos cálidos y húmedos que generan precipitaciones concentrados en más del $90 \%$ en el verano (diciembre-marzo) y el Anticiclón Subtropical del Pacífico Sur, que luego de atravesar la Cordillera y Patagonia, aporta por el sur vientos fríos y secos con heladas ocasionales y precipitaciones reducidas en invierno. Los patrones espaciales de precipitación a escala regional dependen de la topografía. Los sitios húmedos, están localizados en las pendientes orientales de las cadenas montañosas altas. Entre $1000 \mathrm{y}$ 2000 msnm, el rocío por condensación agrega una cantidad significativa de humedad, en especial durante el invierno (Cabrera, 1976; Villalba et al., 1998; Brown et al., 2001; Paolini, 2014).

\section{Actividades humanas}

La actividad humana en el área es intensa y variada; incluye la producción ganadera en el monte, la extracción de madera con poco control y planificación, agricultura a pequeña escala y la producción de gas y petróleo. Estas actividades tienen una importancia ambiental creciente en la región vecina a la ciudad de Tartagal, en la provincia de Salta, en un contexto de alta demanda social.

\section{MATERIAL Y MÉTODOS}

\section{Palinología, geoquímica y mineralogía}

Se extrajo un testigo de $39 \mathrm{~cm}$ de longitud del borde de la laguna, mediante un muestreador tipo Beeker, que fue sellado en el campo para evitar pérdida de agua y posibles perturbaciones. Una vez, en laboratorio se procedió a la apertura longitudinal del testigo y, en una primera instancia, se tomaron fotografías del mismo mediante una cámara CCD de alta resolución. Se llevaron a cabo estudios palinológicos con muestreos a intervalos de 1 $\mathrm{cm}$. El procesamiento de polen se realizo mediante la técnica estándar para sedimentos cuaternarios (Gray, 1965; Faegri \& Iversen, 1989), la cual consiste en el filtrado con una malla de $250 \mu \mathrm{m}$, incorporación de 2 pastillas de Lycopodium clavatum como marcador foráneo, tratamiento con $\mathrm{KOH}$ al $10 \%$ para la eliminación de la materia orgánica, tratamiento con HF para la eliminación del sílice, filtrado, acetólisis, lavado con acido acético y montaje de muestras. Los conteos (excluyendo esporas de pteridofitas y hongos) se realizaron con un microscopio LEICA ICC 50 con un aumento de 40x, (mínimo 300 granos de polen por muestra) y la identificación sistemática de los tipos polínicos a nivel de especie, género y familia botánica, se realizaron empleando la palinoteca de referencia del Laboratorio de Palinología de la Facultad de Ciencias Agrarias de la Universidad Nacional de Jujuy (PAL-JUA) y los atlas polínicos de Heusser (1971) y Markgraf \& D'Antoni (1978). Para la nomenclatura de los tipos polínicos se siguió las indicaciones de Joosten \& De Klerk (2002) y De Klerk \& Joosten (2007).

La cuantificación de los diversos elementos químicos, se llevo a cabo a través de estudios geoquímicos cada $3 \mathrm{~mm}$ por fluorescencia de rayos-X con un scanner de testigos tipo Avaatech. El generador de XRF utilizó un tubo de Rodio 17 a 10 y $30 \mathrm{kV}$ para producir la excitación de los electrones de los diferentes elementos químicos presentes. Las condiciones de medición fueron las siguientes: amperaje: $2000 \mu \mathrm{A}$, a) voltaje $10 \mathrm{kV}$ con tiempo de exposición de $10 \mathrm{~s}$ para los elementos $\mathrm{Al}, \mathrm{Si}, \mathrm{S}, \mathrm{K}, \mathrm{Ca}, \mathrm{Ti}$, y Fe y b) voltaje $30 \mathrm{kV}$ con exposición de 30 s para los elementos $\mathrm{Br}$, Rb, Sr y Zr.

Para los estudios mineralógicos se extrajeron muestras del testigo a intervalos de $1 \mathrm{~cm}$. Las mismas se secaron en estufa a $60 \mathrm{C}^{\circ}$ durante 24 horas, y se molturaron manualmente en un mortero de ágata. El análisis mineralógico se efectuó mediante un difractómetro de rayos-X automático Siemens D-500, bajo las siguientes condiciones: $\mathrm{Cu} \mathrm{k} \alpha, 40 \mathrm{kV}, 30 \mathrm{~mA}$ y monocromador de grafito. La identificación de las especies minerales y su cuantificación se realizó utilizando técnicas estándares según Chung (1974).

La cronología de alta resolución del testigo se basó en el análisis de 12 submuestras por ${ }^{210} \mathrm{~Pb}$ realizados en el laboratorio de Environmental Radioactivity Research Centre University of Liverpool.

Se emplearon métodos numéricos multivariados para la interpretación de los datos obtenidos, mediante el software R (2012). Se realizó la ordenación mediante análisis de redundancias (RDA) y de componentes principales (PCA); los cálculos de los porcentajes polínicos y los gráficos se efectuaron con el programa TILIA 2.0.41 de Grimm (2015). 


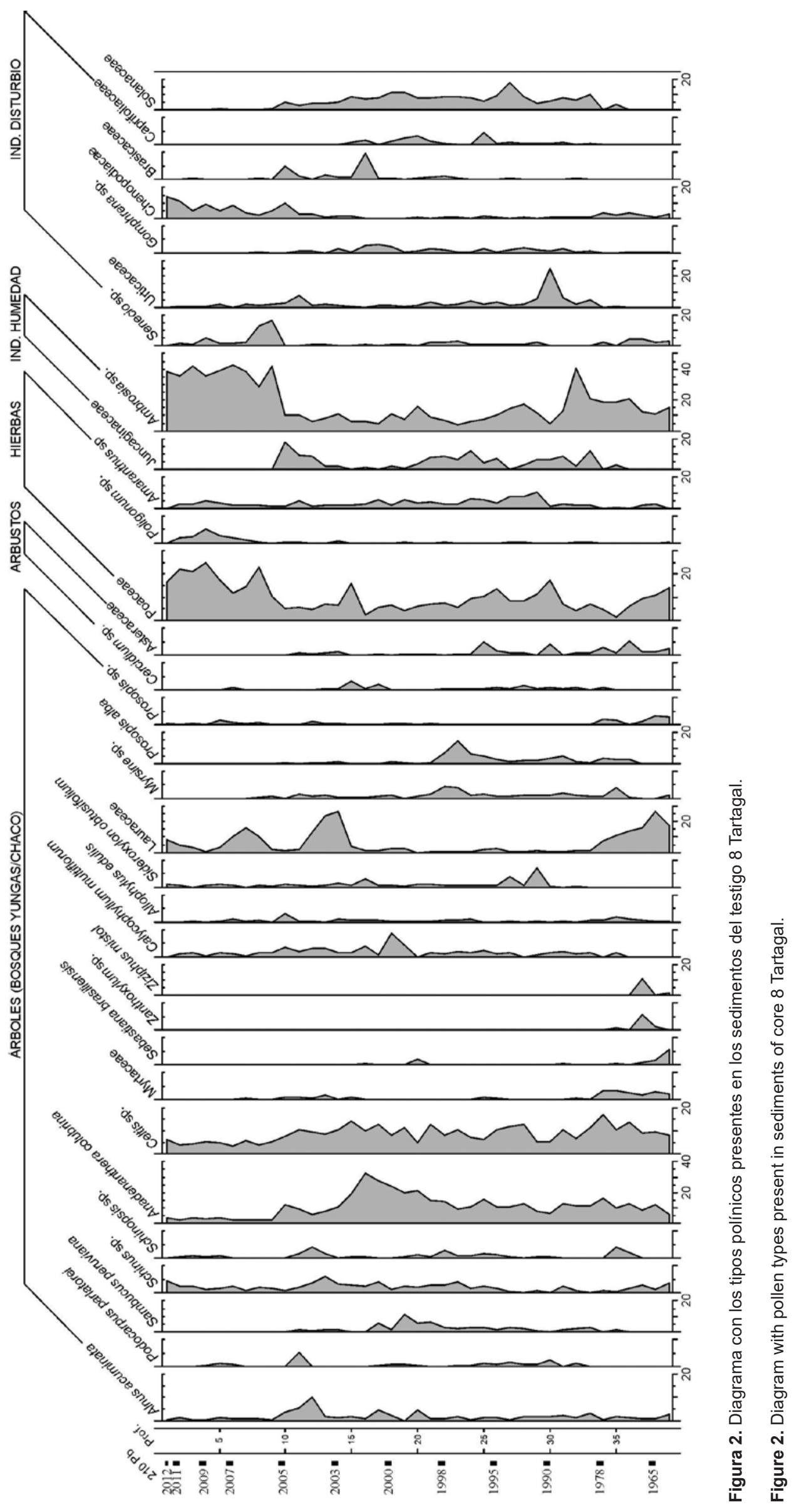




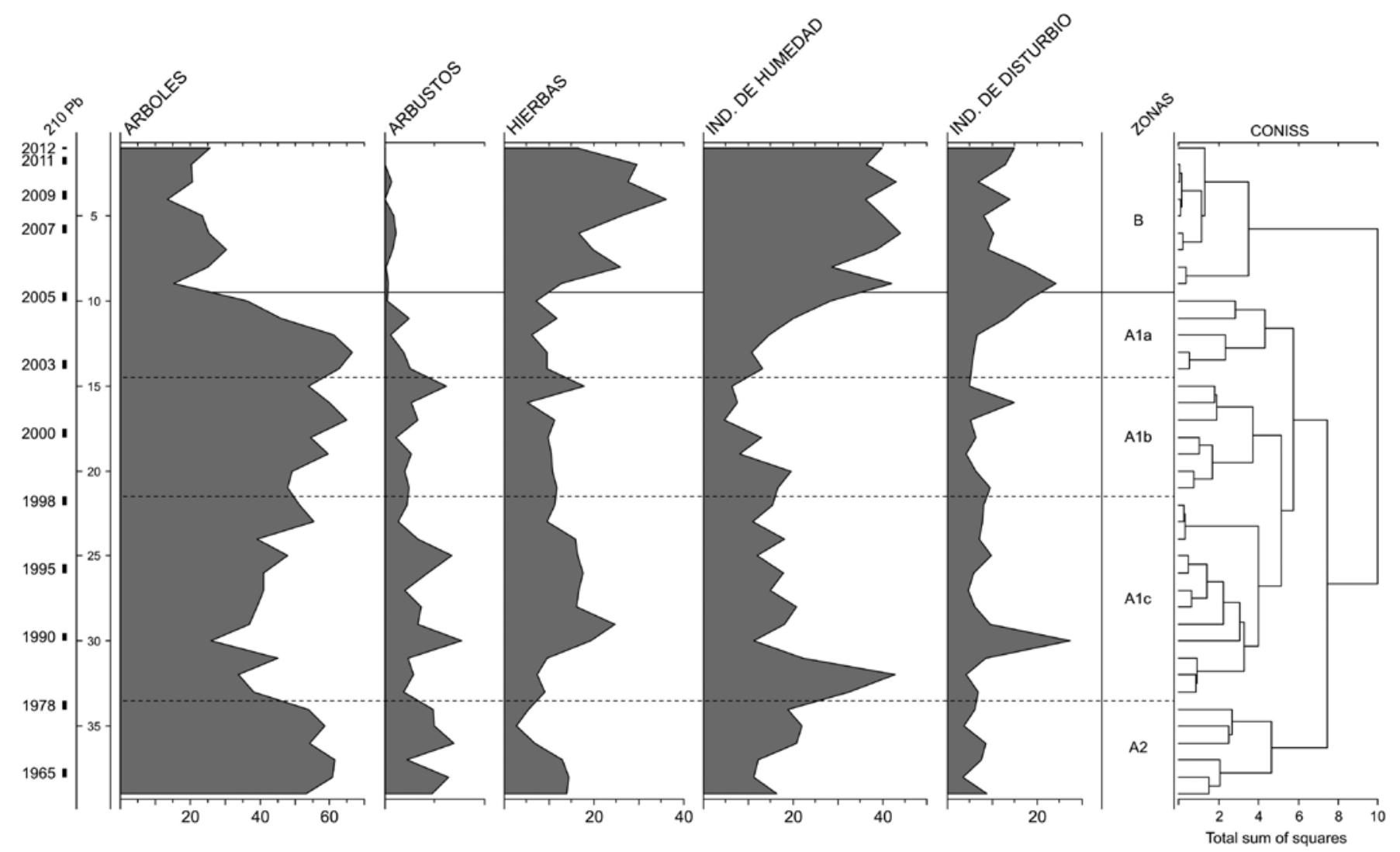

Figura 3. Diagrama sintetizado de las asociaciones polinicas y zonación de análisis cluster del Testigo 8 Tartagal.

Figure 3. Synthesized diagram of pollen associations and zonation of cluster analysis of Core 8 Tartagal.

Los datos de precipitación anual del periodo 1959-2012, se obtuvieron de la base de datos del Servicio Meteorológico Nacional.

\section{RESULTADOS Y DISCUSIÓN}

\section{Palinología}

Las Figuras 2-3 presentan el espectro polínico y la zonación según análisis cluster del testigo 8 Tartagal. Los tipos arbóreos están representados en todo el registro sedimentario con valores altos, entre 15 y $65 \%$, seguido de los indicadores de humedad local (7-45\%), mientras que el resto de los tipos polínicos (arbustos, hierbas e indicadores de disturbio) poseen porcentajes menores al $30 \%$.

Los tipos arbóreos encontrados corresponden a especies de los distintos pisos de Yungas, representados por elementos de Bosque Montano como Alnus acuminata (Figura 4A), Podocarpus parlatorei, Sambucus peruviana y Schinus sp., de Selva Montana como Calycophyllum multiflorum, de Selva Pedemontana como Anadenanthera colubrina (Figura 4C), presente en todo el registro y que, junto a Tipuana tipa (no encontrado en el sedimento), constituyen las especies dominantes en los filos y sectores más secos de la Selva Pedemontana. Estas son especies pioneras en la sucesión de vegetación, intolerantes a la sombra y generalmente dispersadas por el viento (Grau, 2004; Minnetti et al., 2009). También están presentes elementos que se distribuyen en los tres pisos de Yungas como Allophylus edulis (Figura 4D). Los elementos chaqueños y de transición ChacoSelva Pedemontana, como Prosopis, están escasamente representados.

La asociación Ambrosia sp. (Figura 4K) herbácea observada en todo el registro y Juncaginaceae, se vincula a los indicadores de humedad del borde de la laguna. Los indicadores de disturbio relacionados a la presencia de ganado están representados por Senecio sp., Gomphrena sp., Urticaceae, Chenopodiaceae y Brassicaceae (Figura 4) (Braun Wilke, 1995; Lupo et al., 2006; Braun Wilke et al., 2013).

Los tipos polínicos de importancia forestal, como Calycophyllum multiflorum y Phillostilon rhamnoides, aparecen en el registro en bajos porcentajes; esta última especie se encuentra con valores menores a $3 \%$ y posiblemente se deba a la explotación selectiva al que fueron sometidas hasta 1980 aproximadamente (Figura 3 Zona A2), actividad que posteriormente fue declinando o bien a la biología reproductiva relacionada al tipo de polinización anemófila. Es posible que la ausencia en el registro sedimentario, de los tipos polínicos de especies forestalmente valiosas, como Cedrela balansae (polinización entomófila), Tabebuia impetiginosa, Amburana cearensis, Astronium urundeuva (anemófilas), que están presentes en el área de estudio, sea debido a los altos niveles de cosecha y a la modalidad de extracción de los árboles semilleros; 

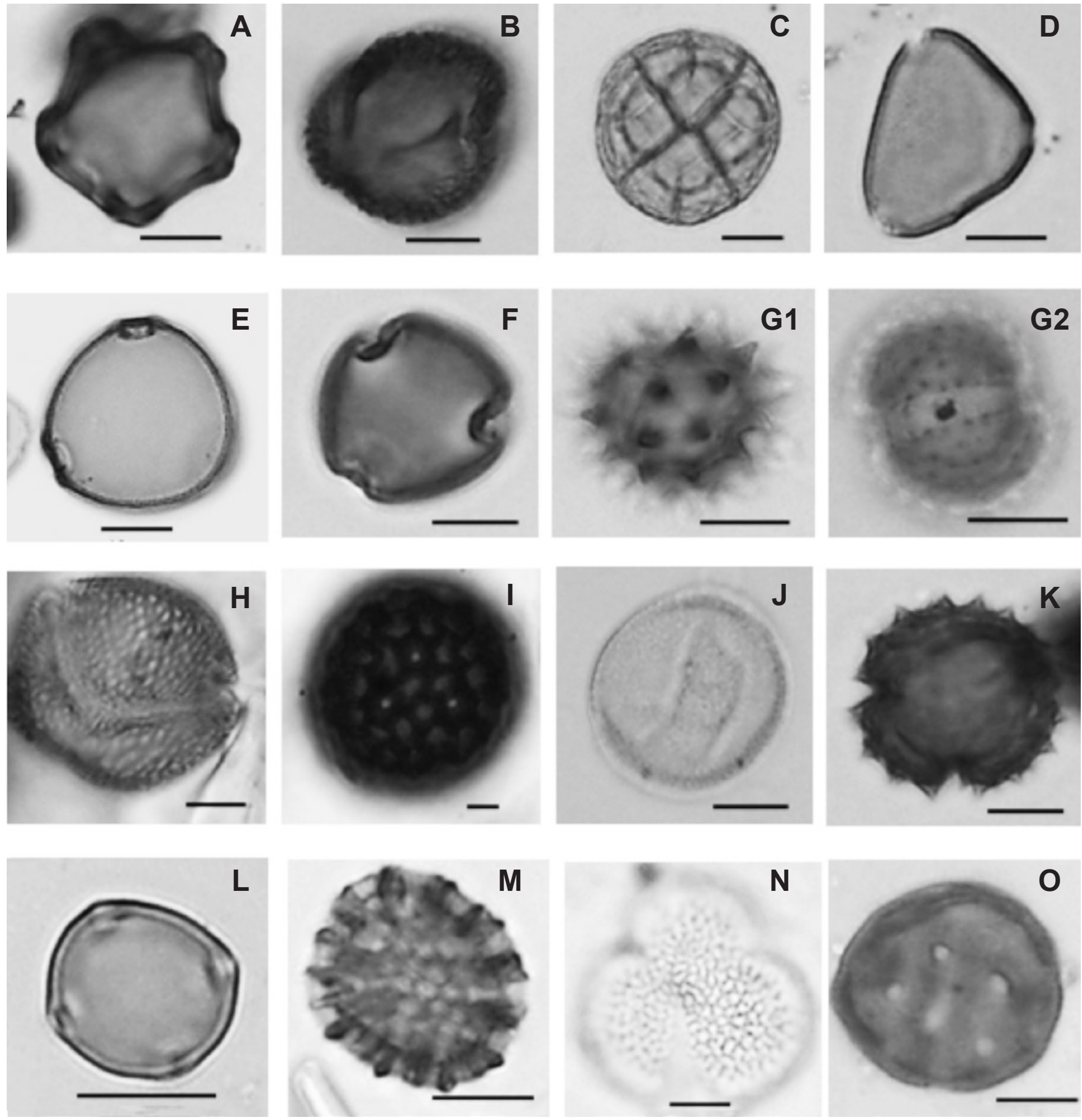

Figura 4. Tipos polínicos presentes en la secuencia sedimentaria del Testigo 8 Tartagal. A, Alnus acuminata Kunth. B, Schinopsis sp. C, Anadenanthera colubrina (Vell.) Brenan. D, Allophylus edulis (A. St.-Hil., A. Juss. \& Cambess.) Radlk. E, Celtis sp. F, Prosopis alba Griseb. G1-G2, Asteraceae. H, Cercidium sp. I, Polygonum sp. J, Juncaginaceae. K, Ambrosia sp. L, Urticaceae. M, Gomphrena sp. N, Brasicaceae. $\mathbf{O}$, Chenopodiaceae. Escalas $=10 \mu \mathrm{m}$.

Figure 4. Pollen types present in the sedimentary sequence of Core 8 Tartagal. Scale bars $=10 \mu \mathrm{m}$.

lo cual implicaría, de acuerdo a Minetti et al. (2009), un progresivo empobrecimiento del bosque y una mala sanidad $\mathrm{y}$ forma de los individuos remanentes.

Entre 1962 y 2005 (Figura 3 Zona B: subzonas B2 y B1b) los árboles son los mejores representados en el registro, en tanto los indicadores de humedad muestran un pico a principios de la década del 80 del siglo pasado, para luego disminuir gradualmente hacia el año 2000. Durante los últimos siete años de la secuencia (2005-2012, Figura 3 Zona A), se incrementan las hierbas y los indicadores de humedad local, coincidiendo con el aumento de las precipitaciones anuales, mientras que los árboles muestran una considerable disminución. 


\section{Geoquímica y mineralogía}

Los sedimentos recuperados están compuestos de arcillas y limos rojos masivos, con intercalaciones de láminas y capas más oscuras de sedimentos arcillosos, ricos en materia orgánica. En la parte inferior de este registro, se observan macrorestos vegetales fibrosos.

Las dataciones por radiocarbono y ${ }^{210} \mathrm{~Pb}$ indican que el registro sedimentario cubre el lapso 1959 y 2012 (Tabla 1). Se observa un marcado incremento de la tasa de sedimentación a partir de la década del '90 (Figura 5), concomitante con la mayor presencia de sedimentos limoarcillosos rojos (clásticos) respecto al tramo inferior, donde predominan los sedimentos ricos en materia orgánica.

Se analizó la mineralogía de 39 muestras, cuya constitución promedio es de $55 \%$ de cuarzo y $23 \%$ de illita acompañados por clinoclorita, caolinita y albita con valores promedio entre 6 y $8 \%$ (Figura 6); las variables de los datos geoquímicos fueron Al, Si, K, Ti, Mn, Fe, Ca, Co, Cr, Rb, Sr y Zr (Figura 7), asociados a las arcillas.

Tabla 1. Cronología por ${ }^{210} \mathrm{~Pb}$ del Testigo 8 de la Laguna Seca de Tartagal.

Table 1. ${ }^{210} \mathrm{~Pb}$ chronology of the Laguna Seca Tartagal Sediment Core 8.

\begin{tabular}{cccccccc}
\hline \multicolumn{2}{c}{ Prof. (cm) } & \multicolumn{2}{c}{ Cronología } & \multicolumn{3}{c}{ Tasa de Sedimentación } \\
\hline $\mathrm{cm}$ & $\mathrm{g} \mathrm{cm}^{-2}$ & $\begin{array}{c}\text { Años } \\
\text { AD }\end{array}$ & $\begin{array}{c}\text { Edad } \\
\text { A }\end{array}$ & +- & $\mathrm{g} \mathrm{cm}^{-2} \mathrm{a}^{-1}$ & $\mathrm{Cm} \mathrm{y}^{-1}$ & + +- (\%) \\
\hline 0,0 & 0,0 & 2012 & 0 & 0 & & & \\
1,5 & 2,2 & 2011 & 1 & 1 & 1,74 & 1,20 & 23,8 \\
3,5 & 5,2 & 2009 & 3 & 2 & 1,74 & 1,32 & 23,8 \\
5,5 & 8,0 & 2007 & 5 & 2 & 1,74 & 1,54 & 23,8 \\
9,5 & 12,5 & 2005 & 7 & 3 & 1,74 & 1,62 & 23,8 \\
13,5 & 15,8 & 2003 & 9 & 3 & 1,74 & 1,64 & 23,8 \\
17,5 & 20,2 & 2000 & 12 & 4 & 1,74 & 1,62 & 23,8 \\
21,5 & 24,9 & 1998 & 14 & 4 & 1,74 & 1,28 & 23,8 \\
25,5 & 29,7 & 1995 & 17 & 5 & 1,74 & 0,70 & 23,8 \\
29,5 & 34,3 & 1990 & 22 & 5 & 0,32 & 0,48 & 19,2 \\
$33-5$ & 28,4 & 1978 & 32 & 8 & 0,32 & 0,40 & 19,2 \\
37,5 & 42,5 & 1965 & 47 & 13 & 0,32 & 0,31 & 19,2 \\
\hline
\end{tabular}

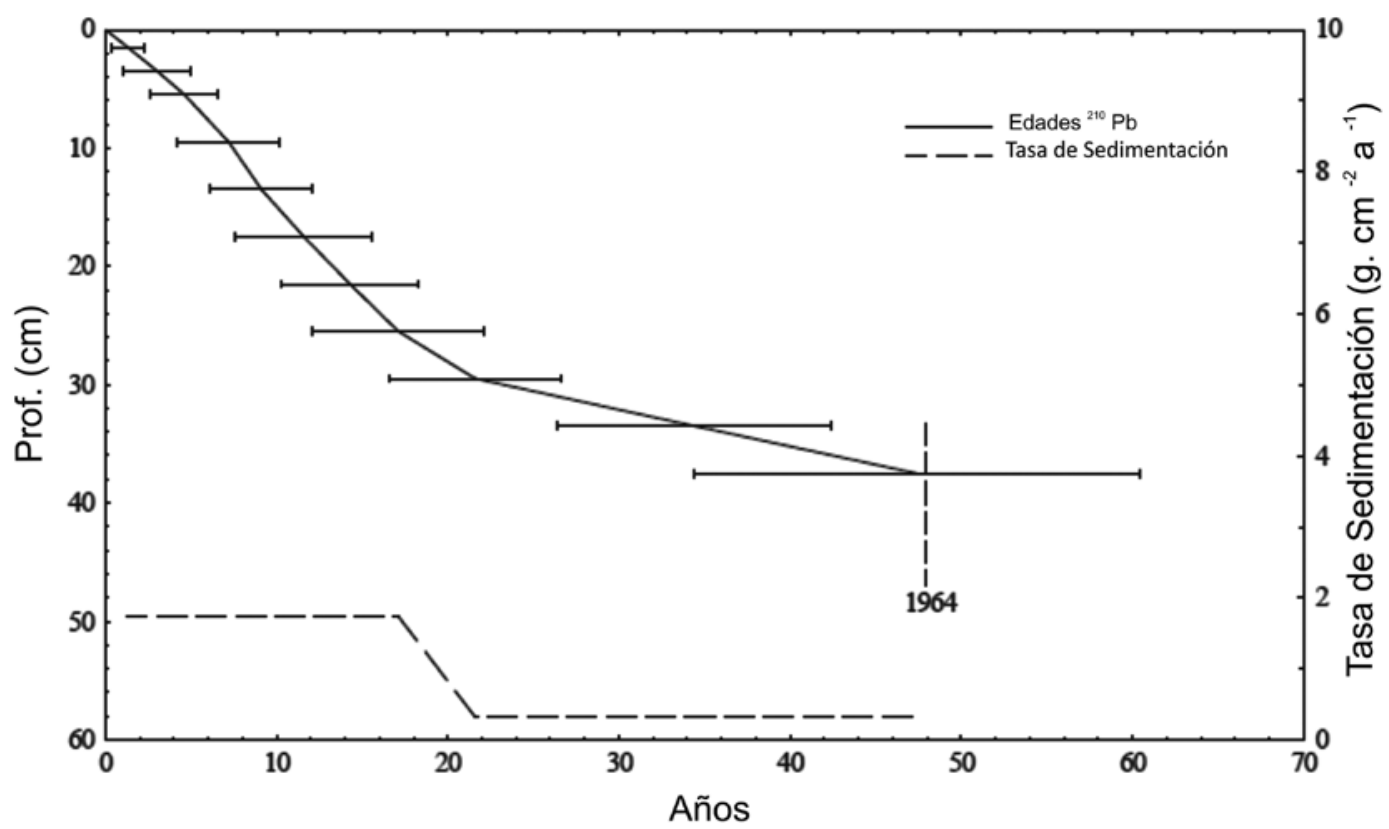

Figura 5. Cronología radiométrica de los sedimentos del testigo 8 de la Laguna Seca de Tartagal mostrando los fechados por ${ }^{210} \mathrm{~Pb}$ y tasas de sedimentación.

Figure 5. Radiometric chronology of the Laguna Seca Tartagal Sediment Core 8 showing the ${ }^{210} \mathrm{~Pb}$ dates and sedimentation rates. 


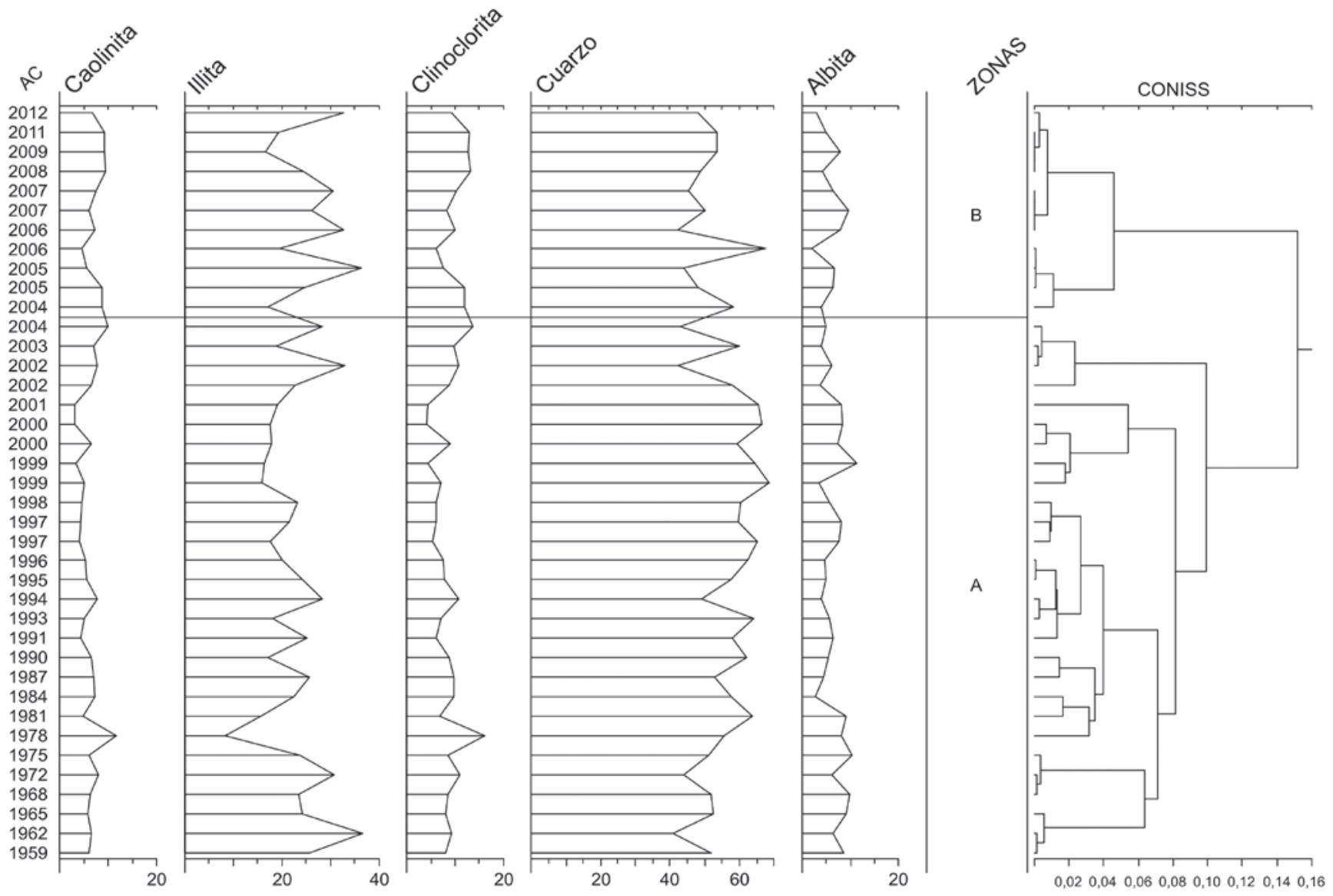

Figura 6. Composición mineralógica y zonación de los sedimentos del Testigo 8 Tartagal.

Figure 6. Mineralogical composition and zonation of the sediments of Core 8 Tartagal.

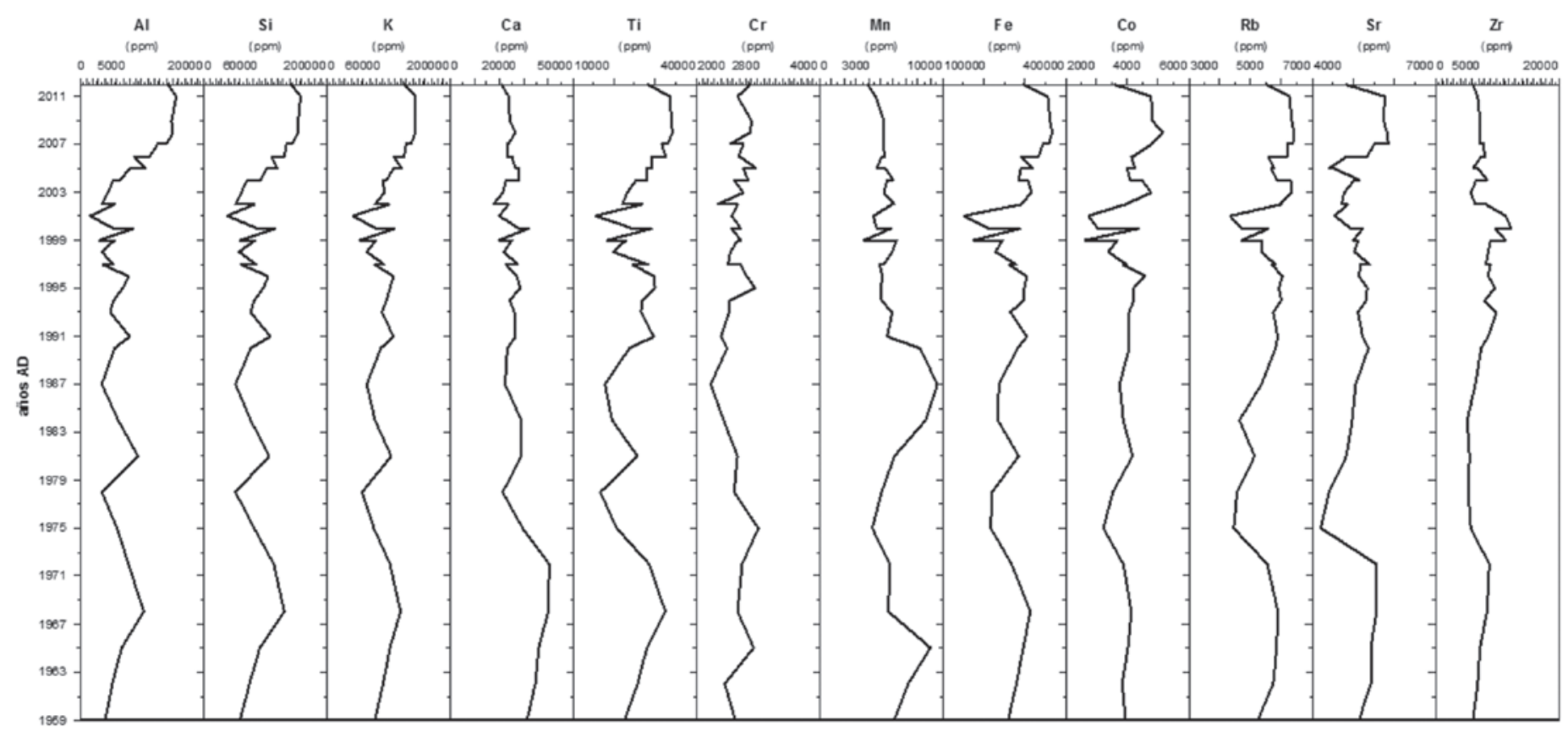

Figura 7. Composicion geoquimica de los sedimentos del Testigo 8 Tartagal.

Figure 7. Geoquimical composition of the sediments of Core 8 Tartagal. 
El análisis de componentes principales (PCA) sobre los datos de XRF del testigo 8 Tartagal (Figura 8A), muestra que el primer vector propio (EV1) está controlado por la mayor parte de elementos químicos en la parte negativa del vector, mientras que el segundo vector propio (EV2) contrapone el Mn (extremo positivo) y el $\mathrm{Zr}$ y Cr (extremo negativo).
La mayor parte de los elementos químicos se relaciona a minerales terrígenos, tanto gruesos (cuarzo) como finos (albita, illita), mientras que el $\mathrm{Mn}$ se asocia a minerales de alteración (caolinita, clinoclorita) (Figura 8B). La entrada de terrígenos a la laguna, se vincula a eventos de precipitación intensa (año 1984, periodo 1996-1997, 2000-2009), responsables de la erosión de las vertientes en su cuenca de drenaje.
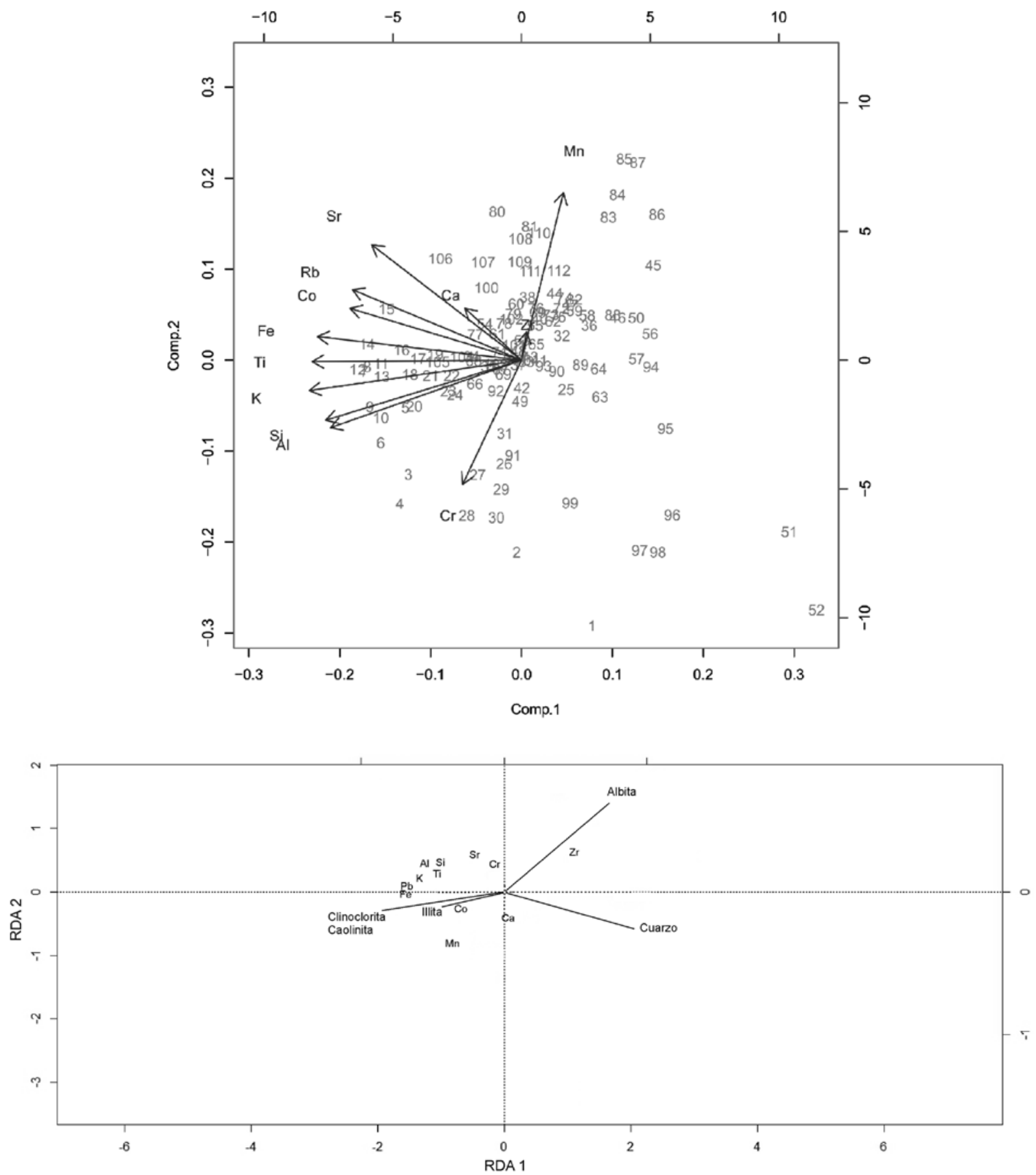

Figura 8. A, Análisis de Redundancia (RDA) utilizando los datos geoquímicos remuestreados a 3 mm constreñidos con los datos mineralógicos. $\mathrm{B}$, análisis de componentes principales (PCA), del Testigo 8 Tartagal.

Figure 8. A, Redundancy Analysis (RDA) using geochemical data resampled to $3 \mathrm{~mm}$ constrained with mineralogical data. B, Principal component analysis (PCA) of Core 8 Tartagal. 
Las anomalías climáticas vinculadas al fenómeno de "El Niño", especialmente a partir del evento ocurrido en 1982/83 y el posterior de 1997/98, coincidieron con las inundaciones a nivel regional del Litoral y Chaco Argentino (Caputo et al., 1998) y con el aumento de ocurrencia de deslizamientos de laderas en el departamento San Martin en el periodo 1999-2005 (Cristobal et al., 2009).

Durante los periodos de baja intensidad de precipitación (años 1962, 1969, periodos 1974-1979, 1982-1983, 19851986), la sedimentación es rica en materia orgánica. Por lo tanto, las variaciones del EV1 se consideran como indicativas de fluctuaciones en la entrada de terrígenos, los cuales son, a su vez, marcadores de la intensidad de precipitación.

\section{Interpretación paleoambiental}

Los resultados de la palinología, geoquímica y mineralogía (Figura 9), son consistentes con la precipitación anual en Tartagal durante las últimas décadas y se correlacionan con los fenómenos de aluviones y deslizamientos ocurridos en la Sierra de Tartagal y su piedemonte oriental (Tabla 2), como

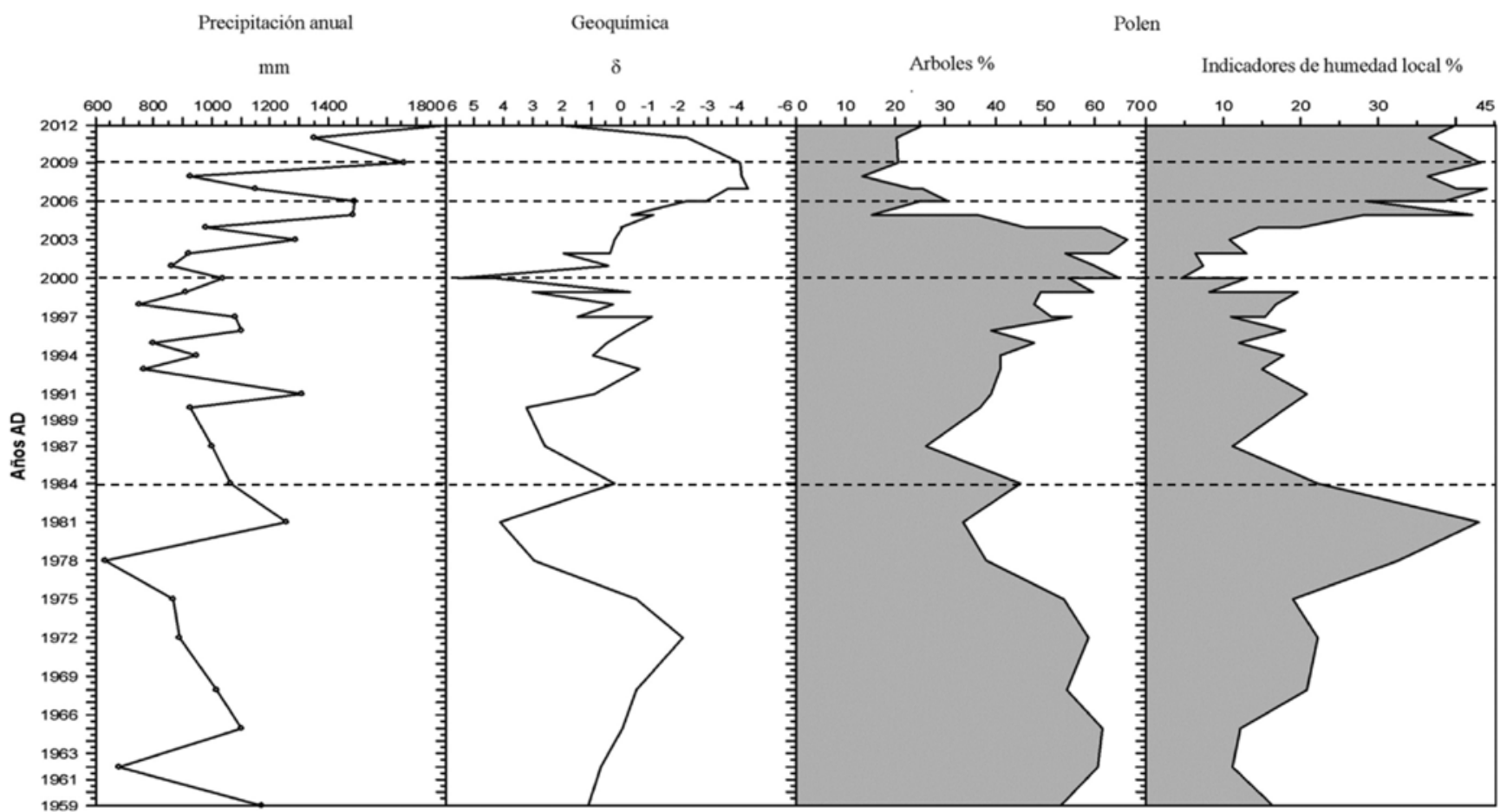

Figura 9. Evolución de la precipitación anual entre 1962 y 2012, reconstrucción cualitativa de la precipitación obtenida a partir del PCA de los datos de XRF y diagrama polínico reducido (árboles e indicadores de humedad) del Testigo 8 Tartagal. Las líneas representan los años más significativos de fenómenos de aluviones y deslizamientos en la Sierra de Tartagal (Tabla 2)

Figure 9. Evolution of annual rainfall between 1962 and 2012, qualitative reconstruction of precipitation obtained from the PCA data XRF, and reduced pollen diagram (trees and humidity indicators) of Core 8 Tartagal. The lines represent the most significant years of alluvial events and landslides in the mountain range of Tartagal (Table 2).

Tabla 2. Cronología de eventos catastróficos ocurridos en el departamento San Martín, Provincia de Salta, República Argentina.

Table 2. Chronology of catastrophic events in the department of San Martín, Salta, Argentina.

\begin{tabular}{clll}
\hline Año & \multicolumn{1}{c}{ Lugar } & Evento & Daños \\
\hline 1984 & Campamento Vespucio & 25 de octubre aluvión & $\begin{array}{l}\text { Infraestructura, nueve personas } \\
\text { fallecidas, evacuados }\end{array}$ \\
2000 & Campamento Vespucio & 22 de octubre aluvión & $\begin{array}{l}\text { En infraestructura, una persona } \\
\text { fallecida, evacuados. }\end{array}$ \\
2006 & Campamento Vespucio y Ciudad de Tartagal & Febrero de 2006 aluvión, inundación & $\begin{array}{l}\text { En infraestructura, personas } \\
\text { fallecidas, evacuados }\end{array}$ \\
2009 & Ciudad de Tartagal & 9 de febrero inundación, aluvión & $\begin{array}{l}\text { Infraestructura, personas fallecidas, } \\
\text { personas evacuadas }\end{array}$ \\
\hline
\end{tabular}


los ocurridos en diciembre de 2005 y enero de 2006 a causa de una sucesión de tormentas de gran intensidad (con valores extremos, considerados record para la zona) que produjeron numerosas muertes, la destrucción de viviendas, infraestructura y anegamientos. Esto fue una combinación de factores naturales (morfología de la cuenca, características geológicas, suelos, precipitaciones, cobertura vegetal) y probablemente también antrópicos, especialmente relacionadas al uso concurrente de los recursos naturales (Cabral \& Plaza, 2006; Baumman et al., 2009; Pacheco \& Cristóbal, 2009; Cardozo, 2013). Diversos estudios a diferentes escalas espaciales y temporales (Veblen \& Asthon, 1978; Garwood et al., 1979; Guariguata, 1990; Blodgett, 1998; Trauth et al., 2003; Remondo et al., 2005; Paolini, 2014) muestran que las precipitaciones, las fuertes pendientes de las zonas montañosas y los cambios en el uso de suelo, juegan un papel importante en la dinámica de los deslizamientos de ladera.

Estas nuevas evidencias, consideradas en el contexto de reconstrucciones del clima basadas en registros instrumentales y dendocronológicos reflejan el substancial aumento en las precipitaciones durante los últimos 50 años en la región del Noroeste Argentino y la respuesta del ambiente (Bianchi \& Yañez, 1992; Villalba, 1995; Brown et al., 2001; Plaza et al., 2006; Morales et al., 2012). El registro de alta sensibilidad ambiental muestra: (i) aumento de la sedimentación de terrígenos y de la tasa de sedimentación desde 1990; (ii) una buena correlación de la geoquímica con las variaciones de las precipitaciones, en particular la tendencia creciente en las lluvias desde 1974, que se vincula a partir de 1984 a la aparición como fenómeno recurrente, de los eventos de remoción en masa que perduran hasta el presente; (iii) la última parte del registro polínico de Laguna Seca muestra un incremento en los niveles de la laguna, evidenciados por los indicadores de humedad local y de las hierbas y la reducción de los tipos polínicos de árboles, como respuesta a una mayor frecuencia de deslizamientos vinculadas a lluvias intensas y abundantes, así como también, de la rápida aparición de especies colonizadoras (Anadenanthera colubrina). Estos contextos permiten apoyar las evidencias de la intensificación de la circulación atmosférica continental, incrementando las precipitaciones de la región hasta alcanzar los niveles más altos de, al menos, los últimos dos siglos (Minetti \& Vargas 1997; Villalba et al., 1998).

El rol de la presión antrópica para el aprovechamiento de los recursos naturales que desencadena e intensifica la frecuencia de los episodios de remoción en masa (Brown,1995; Castro, 1995; Prado, 1995; Gonzalez Bonorino et al., 2001; Remondo et al., 2005; Grau et al., 2005; Cristóbal et al., 2009; Pacheco \& Cristóbal, 2009; Busnelli, 2012), podrá ser mejor discutido a través de las herramientas de reconstrucción aquí empleadas, en la medida en que se logren obtener secuencias con una profundidad temporal mayor a 100 años, es decir, que nos permitan comprender el estado del ambiente en momentos que consideramos previos a los mayores impactos humanos en el área de estudio, para poder precisar las diferentes evidencias naturales y antrópicas de cambios ambientales.

\section{CONCLUSIONES}

El estudio palinológico, geoquímico y mineralógico de detalle de un testigo sedimentario en la Laguna Seca de Tartagal, ha permitido la reconstrucción del paisaje y de las variaciones de precipitación para el periodo 1959-2012 en las Sierras Subandinas del norte de Salta. Los distintos proxies utilizados (polen, geoquímica y mineralogía) reflejaron con sensibilidad diferencial los cambios ambientales, tales como las precipitaciones, el estado de la cobertura vegetal y los niveles relativos de la laguna.

El aumento en las precipitaciones a partir de 1974 y la posterior intensificación, a partir de 1984, de eventos recurrentes de remoción en masa en la Sierra de Tartagal, es una tendencia que perdura hasta el presente, con grave afectación a la población e infraestructura. Esta fase, con precipitaciones por encima del promedio y lluvias de gran intensidad, así como probablemente, la gestión no integrada de los recursos naturales en la cuenca alta del río Tartagal, constituye el marco de condiciones actuales, de alta fragilidad ambiental, que hace necesario coordinar y maximizar los esfuerzos para el ordenamiento del territorio.

Se destaca la relevancia de estos archivos para la reconstrucción de la historia ambiental, con énfasis en los eventos potencialmente catastróficos para la población ubicada en el piedemonte, considerando que, una futura ampliación de los rangos temporales estudiados así como el aporte de información social y ambiental multitemporal, permitirá incrementar las certezas respecto a la causalidad y magnitud de los procesos catastróficos históricos en el área de Tartagal, en vistas a mejorar la prevención y preparación ante eventos que ponen en riesgo a la infraestructura y a las personas.

La integración de datos multiproxies: palinológicos, geoquímicos y mineralógicos de resolución anual, con datos climáticos, referencias históricas y procesos geomorfológicos activos, ha permitido generar un marco de referencia paleoambiental que contextualiza la dinámica de las comunidades vegetales en Los Bosques Andinos Subtropicales de las Yungas, en un lapso de seis décadas. Este trabajo sienta las bases para reconstruir las variaciones de la vegetación, el clima y la historia del disturbio antrópico durante el Holoceno en las Sierras Subandinas del Noroeste Argentino.

\section{AGRADECIMIENTOS}

Los resultados aquí presentados corresponden a una etapa de realización de tesis de doctorado del primer autor en el marco del Proyecto Picto UNJu 147 "Catástrofes ambiéntales en Puna, Yungas y Chaco". A G. Cortes, G. Torres y E. Pereira por su colaboración en tareas realizadas en campo. A M.J. Rubio y G.S. López por el acompañamiento y asesoramiento técnico en el procesamiento de las muestras con el XRF Y DRX en el ICTJA. A M. Burgos por el procesamiento de muestras palinológicas. A R.B. Wilke y M. Cruz por su ayuda en la traducción de los resúmenes. 


\section{REFERENCIAS}

Argollo, J. \& Mourguiart, P. 2000. Late Quaternary climate history of the Bolivian Altiplano. Quaternary International, 72:37-51. doi:10.1016/S1040-6182(00)00019-7

Baumann, V.; Seggiaro, R.; Azcurra, D. \& García, V. 2009. Inundación de detritos en la ciudad de Tartagal, Salta. Buenos Aires, Instituto de Geología y Recursos Minerales, Servicio Geológico Minero Argentino, 26 p. (Serie Contribuciones Técnicas "Peligrosidad Geológica" 15).

Behling, E. 2011. Holocene environmental dynamics in coastal, eastern and central Amazonia and the role of the Atlantic sea level change. Geographica Helvetica, 66:208-216. doi:10.5194/ gh-66-208-2011

Bianchi, A. \& Yañez, C. 1992. Las precipitaciones del noroeste argentino. Salta, Instituto Nacional de Tecnologia Agropecuaria. $383 \mathrm{p}$.

Blodgett, T.A. 1998. Erosion rate on the NE escarpment of the Eastern Cordillera, Bolivia Derived from Aerial Photographs and Thematic Mapper Images. Cornell University, Tesis doctoral, 149 p.

Blundo, C. \& Malizia, L. 2009. Impacto del aprovechamiento forestal en la estructura y diversidad de la Selva Pedemontana. In: A. Brown; P. Blendinger.; T. Lomascolo \& P. García Bes (eds.) Selva Pedemontana de las Yungas. Historia natural, ecología y manejo de un ecosistema en peligro, Ediciones del Subtrópico, p. 387-405.

Bonachea, J.; Bruschi, V.M.; Hurtado, M.A.; Forte, L.M.; Da Silva, M.; Etcheverry, R.; Cavallotto, J.L.; Dantas, M.F.; Pejon, O.J.; Zuquette, L.V.; De O. Bezerra, M. A.; Remondo, J.; Rivas, V.; Gómez Arozamena, J.; Fernández, G. \& Cendrero, A. 2010. Natural and human forcing in recent geomorphic change; case studies in the Río de la Plata basin. Science of the Total Environment, 408:2674-2695. doi:10.1016/j. scitotenv.2010.03.004

Braun Wilke, R. 1995. Plantas de interes ganadero de Jujuy y Salta Noroeste Argentino. Jujuy, Universidad Nacional de Jujuy, 309 p.

Braun Wilke, R.; Santos, E.E.; Picchetti, L.P.; Larran, M.T.; Guzmán, G.F.; Colarich, C.R. \& Casoli, C.A. 2013. Carta de aptitud ambiental de la Provincia de Jujuy. $2^{\mathrm{a}}$ ed. Jujuy, Ediunju, 269 p.

Brown, A. 1995. Las selvas de montaña del noroeste de Argentina: problemas ambientales e importancia de su conservación. In: A.D. Brown \& H.R. Grau (eds.) Investigación, conservación y desarrollo de las selvas subtropicales de montaña, Laboratorio de Investigaciones Ecológicas de las Yungas, p 9-18.

Brown, A; Grau, H.; Malizia, L. \& Grau, A. 2001. Argentina. In: M. Kappelle \& A. D. Brown (eds.) Bosques nublados del Neotrópico, Editorial IMBIO, p. 623-698.

Burbridge, R.E.; Mayle, F.E. \& Killeenb, T.J. 2004. Fifty thousand year vegetation and climate history of Noel Kempff Mercado National Park, Bolivian Amazon. Quaternary Research, 61: 215-230. doi:10.1016/j.yqres.2003.12.004

Busnelli, J. 2012. Mapa de amenaza de remoción en masa de la provincia de Tucumán según diferentes escenarios de cambio climático. Acta Geológica, 24:4-33.

Cabral, C. \& Plaza, G. 2006. Análisis de la situación global del Municipio de Tartagal, Depto. San Martín. Diagnóstico y evaluación de la cuenca del río Tartagal y área de influencia. 179 p.

Cabrera, A. 1976. Regiones fitogeograficas de la Republica Argentina. In: W.F. Kugler (ed.) Enciclopedia de Agricultura, Jardinera y Fruticultura, Acme, p. 1-85.

Caputo, M.A.; Celis, A.; Gürevich, R.; Herzer, H. \& Petit, H. 1998. Predicción e Imprevisión. Ese es el dilema social. Desastres y Sociedad, 9:21-40.
Cardozo, C. 2013. Zonación de susceptibilidad por procesos de remoción en masa en la cuenca del río Tartagal, Salta (Argentina). Facultad de Matemática, Astronomía y Física e Instituto de Altos Estudios Espaciales Mario Gulich. Universidad Nacional de Córdoba, Tesis de Maestría, 127 p.

Castro, H. 1995. Una aproximación al estudio de las formas de ocupación y uso productivo del sector pedemontano de Yungas. In: A.D. Brown \& H.R. Grau (eds.) Investigación, conservación y desarrollo de las selvas subtropicales de montaña, Laboratorio de Investigaciones Ecológicas de las Yungas, p. 215-222.

Chung, F. 1974. Quantitative interpretation of X-ray diffraction patterns of mixtures: II. Adiabatic principles of X-ray diffraction analysis of mixtures. Journal of Applied Crystallography, 7:526531. doi:10.1107/S0021889874010387

Cortes, G. 2010. Estudio geoarqueológico preliminar en el yacimiento Agroalfarero de Antumpa Departamento de Humahuaca, provincia de Jujuy. Facultad de Humanidades y Ciencias Sociales, Universidad Nacional de Jujuy, Tesis de grado, $157 \mathrm{p}$.

Cristóbal, L.; Pacheco, S.; Malizia, L. \& Garcia Bes, P. 2009. Dinámica de deslizamientos en la Selva Pedemontana del Departamento San Martín. In: A. Brown; P. Blendinger; T. Lomascolo \& P. García Bes (eds.) Selva Pedemontana de las Yungas. Historia natural, ecología y manejo de un ecosistema en peligro, Ediciones del Subtrópico, p. 333-343.

De Klerk, P. \& Joosten, H. 2007. The difference between pollen types and plant taxa: a plea for clarity and scientific freedom. Quaternary Science Journal, 56:162-171.

Faegri, K. \& Iversen, J. 1989. Textbook of pollen analysis. Caldwell, Blackburn Press, 328 p.

Garwood, N.C.; Janos, D.P. \& Brokaw, N. 1979. Earthquake-caused landslides: A major disturbance to tropical forests. Science, 205:997-999. doi: 10.1126/science.205.4410.997

González Bonorino, G.; Rivelli, R. \& Bartoloni, M. 2001. Hoja Geológica 2363-I Tartagal. Provincia de Salta. Buenos Aires, Servicio Geológico Minero Argentino, Instituto de Geología y Recursos Minerales, $331 \mathrm{p}$.

Grau, H.R. 2004 . Dinámica de bosques en el gradiente altitudinal de las Yungas Argentinas. In: M.Arturi; J. Frangi \& J. Goya (eds.) Ecología y manejo de los bosques nativos de Argentina, Universidad Nacional de La Plata, p. 1-30.

Grau, H.R. \& Brown, A.D. 1995. Los deslizamientos de laderas como condicionantes de la estructura y composicion de la selva subtropical de montaña. In: A.D. Brown \& H.R. Grau (eds). Investigacion, conservacion y desarrollo de las selvas subtropicales de montaña, Laboratorio de Investigaciones Ecologicas de las Yungas, p. 79-84.

Grau, H.R.; Gasparri, N.I. \& Aide, M.T. 2005. Agriculture expansion and deforestation in seasonally dry forests of northwest Argentina. Environmental Conservation, 32:140-148. doi:10.1017/S0376892905002092

Gray, J. 1965. Palynological techniques. In: B. Kummel \& D. Raup (eds.) Handbook of Paleontological techniques, W. E. Freeman. p. 471-587.

Grimm, E. 2015. Tilia Software. Springfield, Illinois State Museum.

Guariguata, M. R. 1990. Landslide disturbance and forest regeneration in the upper Luquillo mountains of Puerto Rico. Journal of Ecology, 78:814-832. doi:10.2307/2260901

Heusser, C.J. 1971. Pollen and Spores of Chile. Tucson, The University of Arizona Press, 167 p. 
Joosten, H. \& De Klerk, P. 2002. What's in a name? Some thoughts on pollen classification, identification, and nomenclature in Quaternary palynology. Review of Palaeobotany and Palynology, 122:29-45. doi:10.1016/S0034-6667(02)00090-8

Kulemeyer, J. 2005. Holozäne Landschaftsentwicklung im Einzugsgebiet des Rio Yavi (Jujuy/Argentina). Fakultät fur Biologie, Chemie und geowissenschaften der Universität Bayreuth, Tesis doctoral, $155 \mathrm{p}$.

Kulemeyer, J. 2013. Los cambios ambientales durante el holoceno superior en el norte Argentino y su relevancia e interacción con la arqueología. Anuario de Arqueología, 5:51-64.

Kulemeyer, J. \& Lupo, L. 1998. Evolución del paisaje bajo influencia antrópica durante el Holoceno Superior. Borde oriental de la Puna, Jujuy, Argentina. Bamberger Geographische Schriften, 15:263-276.

Kulemeyer, J.A.; Lupo, L.; Kulemeyer, J.J. \& Laguna, L. 1999. Desarrollo paleoecológico durante las ocupaciones humanas del precerámico del norte de la Puna, Argentina. Bamberger Geographische Schriften, 19:233-255.

Lupo, L. 1998. Estudio sobre la lluvia polinica actual y la evolución del paisaje a través de la vegetación durante el Holoceno en la cuenca del río Yavi. Borde Oriental de la Puna, Noroeste argentino. Universität Bamberg, Alemania, Tesis doctoral, $87 \mathrm{p}$.

Lupo, L.; Bianchi, M.; Araoz, E.; Grau, R.; Lucas, C.; Kern, R.; Tanner, W.; Camacho, M. \& Grosjean, M. 2006. Climate and human impact of the last 2000 years as recorded in Lagunas de Yala, Province of Jujuy, northwestern Argentina. Quaternary International, 158:30-43. doi:10.1016/j.quaint.2006.05.015

Markgraf, V \& D'Antoni, H. 1978. Pollen Flora of Argentina. Tucson, The University of Arizona Press. 208 p.

May J.-H. 2007. Late Quaternary paleoenvironments of Eastern Bolivia deduced from geomorphological and paleopedological archives. Geographisches Institut der Universität Bern, Tesis doctoral, $172 \mathrm{p}$.

May, J.H. 2011. The Río Parapetí Holocene megafan formation in the southernmost Amazon basin. Geographica Helvetica, 66:193-201. doi:10.5194/gh-66-193-2011

May, J.H.; Argollo, J. \& Veit, H. 2008. Holocene landscape evolution along the Andean piedmont, Bolivian Chaco. Palaeogeography, Palaeoclimatología, Palaeoecology, 260:505-520. doi:10.1016/j. palaeo.2007.12.009

Mayle, F.H. 2011. A review of Holocene rainforest ecotonal dynamics at opposite ends of the Amazon-Bolivia versus Colombia. Geographica Helvetica, 66:202-207. doi:10.5194/ gh-66-202-2011

Minetti, J.; Bessonart, S. \& Balducci, E. 2009. La actividad forestal en la Selva Pedemontana del norte de Salta. In: A. Brown; P. Blendinger; T. Lomascolo \& P. García Bes (eds.) Selva Pedemontana de las Yungas. Historia natural, ecología y manejo de un ecosistema en peligro, Ediciones del Subtrópico, p. 367-386.

Minetti, J.L. \& Vargas, W.M. 1997. Trends and jumps in the annual rainfall in South America, south of the $15^{\circ} \mathrm{S}$. Atmosfera, 11:205-221.

Morales, M.S.; Christie, D.A.; Villalba, R.; Argollo, J.; Pacajes, J.; Silva, J.S.; Alvarez, C.A.; Llancabure, J.C.; \& Soliz Gamboa, C.C. 2012. Precipitation changes in the South American Altiplano since $1300 \mathrm{AD}$ reconstructed by tree-rings. Climate of the Past, 8:653-666. doi:10.5194/cp-8-653-2012

Novillo, M.G. \& Sarandón, R. 2002. Evaluación ambiental regional. Metodologías utilizadas en el estudio regional de riesgos hidrogeológicos del Noroeste Argentino, 27 p. Disponible en http://www.ingenieroambiental.com/4014/evambregi.pdf; accesado en 09/08/2016.

Pacheco, S. \& Cristóbal, L. 2009. Cambio de uso de la tierra y fragmentación en la Reserva de Acambuco. In: A. Brown; P. Blendinger; T. Lomascolo \& P. García Bes (eds.) Selva Pedemontana de las Yungas. Historia natural, ecología y manejo de un ecosistema en peligro, Ediciones del Subtrópico, p. 319-332.

Paolini, L. 2014. Cambio climático y deslizamientos de ladera en las Yungas de Argentina. In: L. Malizia; L. Bergesio \& P. Fierro (eds.) Ambiente y Sociedad en la Comarca de Yala, Ediunju y Ediciones del Subtropico, p. 129-160.

Paolini, L. Sobrino, A. \& Jiménez Muñoz, J. 2001. Detección de deslizamientos de ladera mediante imágenes Landsat TM: el impacto de estos disturbios sobre los bosques subtropicales del noroeste de Argentina. Revista de Teledetección, 18:21-27.

Plaza, G.; Perales, A. \& Cabral, C. 2006. Variabilidad de Precipitaciones en el NOA y el riesgo ambiental. Avances en energías renovables y medio ambiente, 10:87-92.

Prado, D.E. 1995. Selva Pedemontana: contexto regional y lista florística de un ecosistema en peligro. In: A.D. Brown \& H.R. Grau (eds.) Investigacion, conservacion y desarrollo de las selvas subtropicales de montaña, Laboratorio de Investigaciones Ecologicas de las Yungas. p. 19-52.

R. 2012. Development Core Team. 2012. A language and environment for statistical computing. R Foundation for Statistical Computing Vienna, Austria. Disponible en http;//CRAN.R-project.org; accesado en 09/08/2016.

Remondo, J.; Sotob, J.; Gonzalez-Dieza, A.; Diaz de Terana, J.R. \& Cendrero, A. 2005. Human impact on geomorphic processes and hazards in mountain areas in northern Spain. Geomorphology, 66:69-84. doi:10.1016/j.geomorph.2004.09.009

Schäbitz, F.; Lupo, L.; Kulemeyer, J. \& Kulemeyer, J.A. 2001. Variaciones de la vegetación, el clima y la presencia humana en los últimos 15.000 años en el Borde Oriental de la Puna, provincias de Jujuy y Salta, Noroeste Argentino. Ameghiniana, 8: $125-130$

Torres, G.R. 2010. Estudio de los cambios del paisaje a través de las comunidades vegetales de alta montaña en el sector nororiental de la puna jujeña. Facultad de Ciencias Agrarias, Universidad Nacional de Jujuy, Tesis de Grado, 68 p.

Trauth, M.H.; Bookhagen, B.; Marwan, N. \& Strecker, M.R. 2003. Multiple landslide clusters record Quaternary climate changes in the northwestern Argentine Andes. Palaeogeography, Palaeoclimatology, Palaeoecology, 194:109-121. doi:10.1016/ S0031-0182(03)00273-6

Veblen, T.T. \& Ashton, P. 1978. Cathastrophic influences on the vegetation of the valdivian Andes, Chile. Vegetation, 36:149167. doi 10.1007/bf02342598

Villagran, C. 1993. Una interpretación climática del registro palinologico del ultimo ciclo glacial y posglacial en Sudámerica. Bulletin de l'Institut Francais d'Etudes Andines, 22:243-258

Villalba, R. 1995. Estudios dendrocronológicos en la Selva Subtropical de Montaña, Implicaciones para su conservación y desarrollo. In: A.D. Brown \& H.R. Grau (eds.). Investigacion, conservacion y desarrollo de las selvas subtropicales de montaña, Laboratorio de Investigaciones Ecologicas de las Yungas, p. 59-68.

Villalba, R.; Grau, H.R.; Boninsegna, J.A. \& Ripalta, A. 1998. Intensificación de la circulación atmosférica meridional en 
la Región subtropical de América del Sur inferida a partir de registros dendroclimatológicos. Bulletin de l'Institut Francais d'Etudes Andine, 27:565-579.

Yacobaccio, H.; Morales, M.; Solá, P.; Samec, C.; Hoguin, R. \& Oxman, B. 2013. Mid-Holocene occupation of the Dry
Puna in NW Argentina: Evidence from the Hornillos 2 rockshelter. Quaternary International, 307:38-49. doi:10.1016/j. quaint.2012.09.028.

Received in October, 2015; accepted in July, 2016. 
Apéndice 1. Listado de tipos polínicos identificados.

Appendix 1. List of pollen types identified.

\section{Gimnospermas}

Podocarpaceae

Podocarpus parlatorei Pilg. (no ilustrada)

\section{Angiospermas}

Betulaceae

Alnus acuminata Kunth (Figura 4A)

Caprifoliaceae (no ilustrada)

Sambucus peruviana Kunth (no ilustrada)

Anacardiaceae

Schinopsis sp. (Figura 4B)

Fabaceae

Anadenanthera colubrina (Vell.) Brenan (Figura 4C)

Prosopis alba Griseb (Figura 4F)

Prosopis sp. (no ilustrado)

Cercidium sp. (Figura 4H)

Celtidaceae

Celtis sp. (Figura 4E)

Myrtaceae (no ilustrada)

Euphorbiaceae

Sebastiana brasiliensis Spreng (no ilustrado)

Rutaceae

Zanthoxylum sp.

Rhamnaceae

Ziziphus mistol Griseb (no ilustrada)

Rubiaceae

Calycophyllum multiflorum Griseb (no ilustrada)

Sapindaceae

Allophylus edulis (A. St.-Hil., A. Juss. \& Cambess.) Radlk (Figura 4D)

Sapotaceae

Sideroxylon obtusifolium (Roem. \& Schult.) T.D. Penn (no ilustrada)

Lauraceae (no ilustrada)

Myrsinaceae

Myrsine (no ilustrada)

Asteraceae (Figuras 4G1-2)

Ambrosia sp. (Figura 4K)

Senecio sp. (no ilustrada)

Poaceae (no ilustrada)

Polygonaceae

Polygonum sp. (Figura 4I)

Chenopodiaceae (Figura 4O)

Juncaginaceae (Figura 4J)

Urticaceae (Figura 4L)

Amaranthaceae

Amaranthus sp. (no ilustrada)

Gomphrena sp. (Figura 4M)

Brassicaceae (Figura 4N)

Solanaceae (no ilustrada) 